\title{
Significance of the Capacity Recovery Effect in Pouch Lithium-Sulfur Battery Cells
}

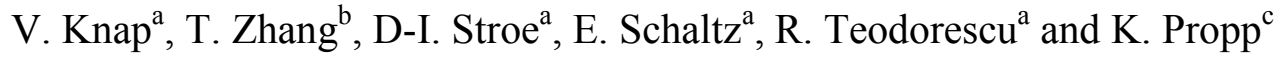 \\ ${ }^{a}$ Department of Energy Technology, Aalborg University, Aalborg, 9000, Denmark \\ ${ }^{b}$ Department of Mechanical Engineering, Imperial College London, SW7 2AZ, United \\ Kingdom \\ ${ }^{\mathrm{c}}$ Centre for Automotive Engineering and Technology, Cranfield University, Bedfordshire \\ MK43 0AL, United Kingdom
}

\begin{abstract}
Lithium-Sulfur (Li-S) batteries are an emerging energy storage technology, which is technically-attractive due to its high theoretical limits; practically, it is expected that Li-S batteries will result into lighter energy storage devices with higher capacities than traditional Lithium-ion batteries. One of the actual disadvantages for this technology is the highly pronounced rate capacity effect, which reduces the available capacity to be discharged when high currents are used. This drawback might be addressed by the use of the capacity recovery effect, which by introducing relaxation periods between consecutive pulse discharges of the battery, increases the available discharge capacity of the cell. The capacity recovery effect of the Li-S cell is studied in this paper using the pulse discharge technique, considering its dependence on the applied current, discharge step length, temperature, and on the length of the relaxation period between the discharging pulses.
\end{abstract}

\section{Introduction}

Nowadays, Lithium-Sulfur (Li-S) batteries are under intensive research and development, as they are characterized by promising high theoretical limits of gravimetric $(2567 \mathrm{Wh} / \mathrm{kg}$ ) and volumetric $(2199 \mathrm{Wh} / \mathrm{l})$ energy densities. The practical reached values of gravimetric energy density at pouch cell level were reported to be around $300 \mathrm{Wh} / \mathrm{kg}$; furthermore, it is expected to reach values of $600 \mathrm{Wh} / \mathrm{kg}$ in the near future (1), (2). However, despite their prospective advantages, Li-S batteries have not been widely commercialized due to their drawbacks, such as high self-discharge rate, short cycle lifetime, and limited rate capability. (3)

The rate capability, or the so-called rate capacity effect, is a general attribute of batteries (4). For Li-S batteries, the limited rate capability is generally believed to be associated with the formation of solid insulating species on the cathode surface. Fan et al (5) recently demonstrated by means of scanning electron microscopy that the surface coverage of the insulating $\mathrm{Li}_{2} \mathrm{~S}$ film on carbon increases with discharge current. As a result of the high $\mathrm{Li}_{2} \mathrm{~S}$ surface coverage, Li-S cells exhibit larger activation over-potential and reduced 
discharge capacity at high currents (6). Precipitation of $\mathrm{Li}_{2} \mathrm{~S}$ could also lead to pore blocking which impedes the ionic transport into the inner cathode. The transport limitation in Li-S batteries can be partially overcome via pulse discharge as shown in (4). Introducing relaxation periods between discharging periods allows the ionic concentrations to equalize across the cell thereby triggering a capacity recovery effect. This capacity recovery effect has been briefly reported for Li-S batteries in (7), (8).

In this paper, the results of a systematic investigation of the capacity recovery effect in a pouch Li-S battery cell are presented. The investigation was performed by applying to the Li-S battery cell various discharging current pulse profiles, in order to determine the dependence of the capacity recovery effect on the applied current, discharge step length, temperature, and on the length of the relaxation period between the discharging pulses.

\section{Experimental}

The experiment was performed on a 3.4 Ah long-life chemistry Li-S pouch cell, manufactured by OXIS Energy. Because a considerable self-discharge of the cell was observed at the high voltage plateau (8), which is caused mainly by the strong presence of the polysulfide shuttle, the experiment was focused on the low voltage plateau. Therefore, this approach allows to observe the charge recovery effect without the influence of the self-discharge, which reduces the total discharge available capacity. The experimental test procedure is illustrated in Fig. 1. At first, a precondition cycle was performed on the cell as follows: charging by 0.1 C-rate until the maximum allowed voltage of $2.45 \mathrm{~V}$ or 11 hours' time limit was reached and then discharging by $0.2 \mathrm{C}$-rate until the minimum allowed voltage of $1.5 \mathrm{~V}$ was reached. Afterwards, the cell was charged by $0.1 \mathrm{C}$-rate to $2.26 \mathrm{~V}$, in order to remain inside the low voltage plateau, and relaxed for four hours to allow the same starting point for all measurements. Discharging steps of a pre-set length (in ampere-hours), followed by pre-defined relaxation periods of various lengths, were repeated until the cut-off voltage of $1.5 \mathrm{~V}$ was reached. The sum of the discharged capacity values measured during the pulses was computed and related to the discharge capacity obtained during the continuous discharge at the same conditions. During the test, the cell was kept in a temperature controlled environment at $25{ }^{\circ} \mathrm{C}$, unless stated otherwise. The test matrix for the considered C-rates, discharging steps lengths and temperatures is presented in Table I.

TABLE I. The test matrix for considered C-rates, discharging steps lengths and temperatures.

\begin{tabular}{cccc}
\hline & \multicolumn{3}{c}{ Discharging step lengths [Ah] } \\
C-rate & 0.05 & 0.1 & 0.2 \\
\hline 0.2 & $\mathrm{X}$ & $\mathrm{X}$ & $\mathrm{X} *$ \\
0.5 & - & - & $\mathrm{X}$ \\
1.0 & - & - & $\mathrm{X}$ \\
\hline
\end{tabular}

*the measurement was performed for 15,25 , and $35^{\circ} \mathrm{C}$ 

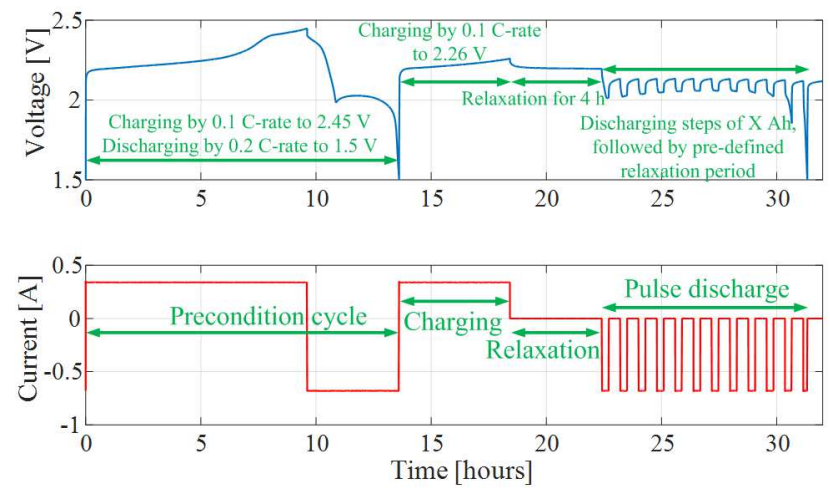

Figure 1. Test procedure of the pulse discharge to capture the capacity recovery effect.

\section{Results and Discussion}

As a base-line, the experiment, which considers the pulse discharge with $0.2 \mathrm{C}$-rate, was performed for the following relaxation period lengths: $0.5,1,5,10,30,60,120,240$ and 480 minutes. The available discharge capacity for the maximum relaxation time of 480 minutes (i.e., 8 hours) was $125.1 \%$ of the capacity measured during continuous discharge at the same conditions. However, this value represents only a minimal increase over the available discharge capacity of $124.6 \%$ measured for a relaxation period of 240 minutes (i.e., 4 hours). Therefore, the relaxation period of 4 hours was considered as a saturation threshold for the measurement of the capacity recovery effect. Thus, the other tests were performed for a reduced number of relaxation periods of $1,5,10,60$ and 240 minutes.

The measured available discharge capacity has an exponential dependency on the relaxation time between pulses. Similar exponential dependencies exist between discharge capacity and other test conditions such as C-rates, discharge pulse lengths and temperatures.

\section{C-rate dependence}

The potential of the Li-S cells' capacity utilization is illustrated, in Fig. 2, by the measured values of available discharge capacity of $124.6,142.8$, and $178.4 \%$ at $0.2,0.5$ and $1 \mathrm{C}$-rate, respectively, for discharge pulses with four hours relaxation period in-between. This indicates that by a proper application design and managing of the Li-S cell, in this case relaxing between discharges (pulse discharge character), the overall discharge time is effectively increased and by that the discharge rate is reduced. Fig. 3 shows a comparison between the absolute capacity values obtained during continuous and pulse discharge with different C-rates. 


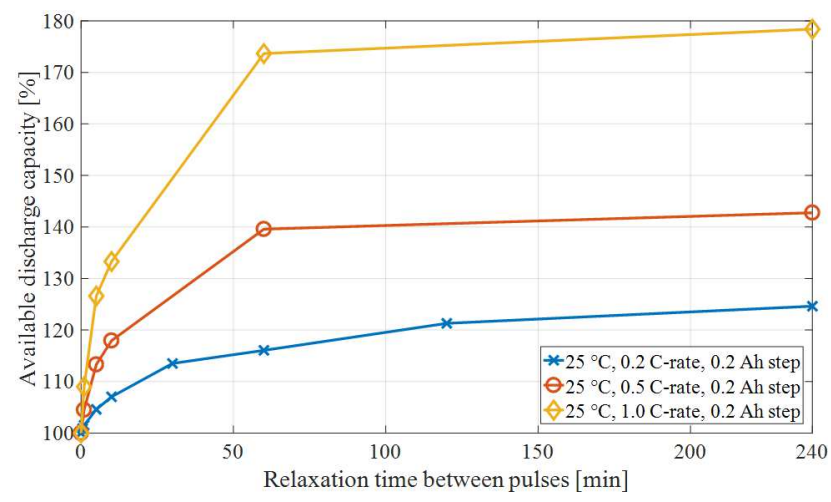

Figure 2. Measured available discharge capacity during the pulse discharge experiments for various C-rates.

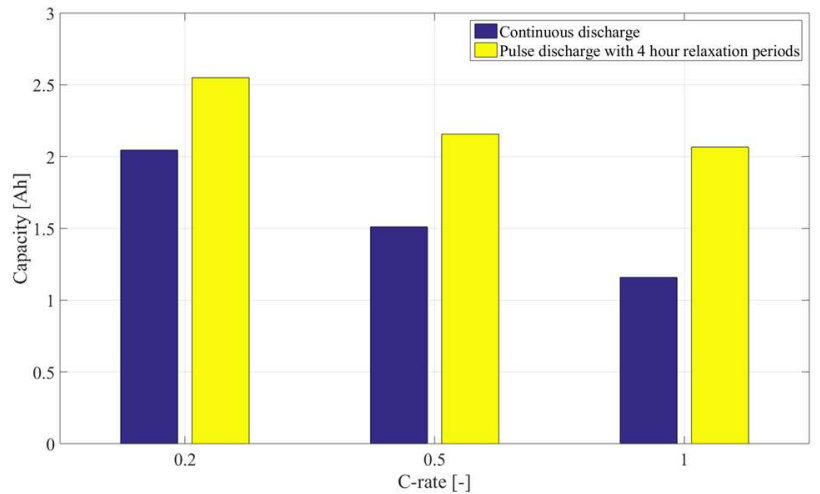

Figure 3. The measured rate capability during continuous discharge and pulse discharge at $25^{\circ} \mathrm{C}, 0.2 \mathrm{C}$-rate and 0.2 Ah step with 4 hour relaxation periods.

\section{Discharging step lengths dependence}

The length of the discharging steps seems to have effect mainly on how fast the capacity is recovered. During a shorter discharge, smaller ionic concentration gradients are formed; consequently, the capacity is recovered faster during the following relaxation period. For the discharge step of $0.05 \mathrm{Ah}$, the cell reached the saturation point already after one hour of relaxation between pulses, while for 0.1 Ah steps, the cell's saturation point was found between one and four hours, which is considerably faster than four hours in the case of 0.2 Ah steps (see Fig. 4). The second, minor, but visible, effect of the various discharge steps is on the amount of recovered capacity. In Fig. 4, there is shown that the recovered capacity with 4 hour relaxation is similar for the cases of 0.1 and $0.2 \mathrm{Ah}$ steps, but in the case of 0.05 Ah steps, the recovered capacity is by $2.3 \%$ higher. 


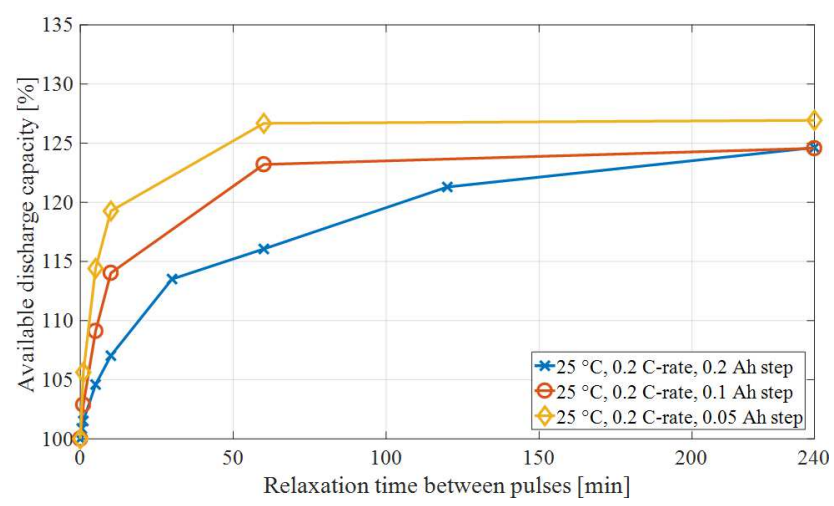

Figure 4. Measured available discharge capacity during the pulse discharge experiments for various discharge step lengths.

\section{Temperature dependence}

With the lower temperature, the cell resistance is higher (9); therefore, the cell reaches the discharge cut-off voltage limit earlier and the absolute discharge capacity is lower. The discharge capacity is further reduced at low temperatures due to the lower ionic diffusion coefficients which generally decrease exponentially with temperature. By allowing the cell for relaxation, the concentration gradients are reduced and the cell's discharge capacity is increased, as shown in Fig. 5. The amount of recoverable capacity through relaxation is larger at lower temperatures due to the more severe transport limitation at low temperatures. The rate of capacity recovery, however, is slower at low temperatures due to the slow ion re-equilibration through diffusion during relaxation.

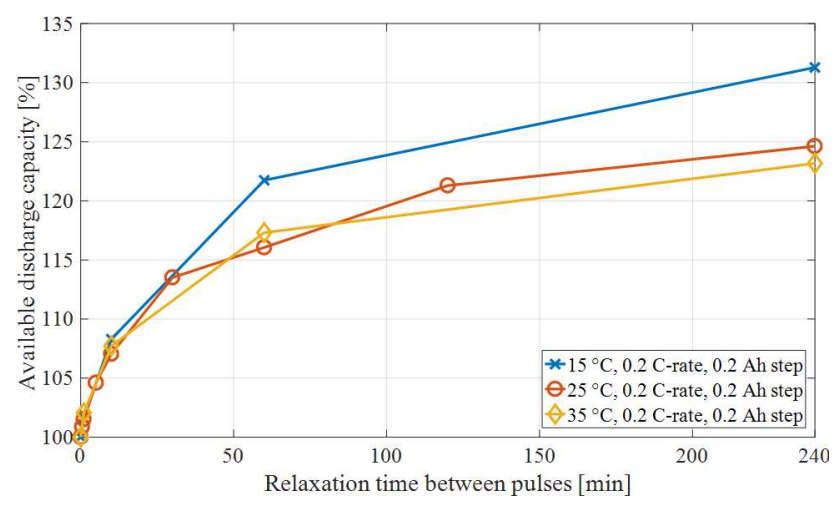

Figure 5. Measured available discharge capacity during the pulse discharge experiments for various temperatures.

\section{Conclusions}

The capacity recovery effect of the Li-S cells was studied in this paper and its significance for the possible practical applications was shown. A high recovery capacity was reached (more than $20 \%$ above the capacity obtained during the continuous discharge) and 
the saturation point for this phenomenon was identified to be between one and four hours for different conditions. It is important to point out that the pulse discharge tests were done at the low voltage plateau of the Li-S cell.

\section{Acknowledgments}

This work has been part of the ACEMU-project. The authors gratefully acknowledge the Danish Council for Strategic Research (1313-00004B) and EUDP (1440-0007) for providing financial support and would like to thank OXIS Energy for supplying the Lithium-Sulfur battery cells.

\section{References}

1. OXIS Energy, http://www.oxisenergy.com/(2016).

2. Sion Power, http://www.sionpower.com/(2016).

3. P. G. Bruce, S. A. Freunberger, L. J. Hardwick, and J.-M. Tarascon, Nat. Mater., 11, 19-29 (2012).

4. V. Rao, G. Singhal, A. Kumar, and N. Navet, VLSI Des. 2005. 18th Int. Conf., 105-110 (2005).

5. F. Y. Fan, W. C. Carter, and Y.-M. Chiang, Adv. Mater, 27, 5203-5209 (2015).

6. S.-E. Cheon, K.-S. Ko, J.-H. Cho, S.-W. Kim, E.-Y. Chin, and H.-T. Kim, J. Electrochem. Soc., 150, A800-A805 (2003).

7. C. E. Parfitt, thesis, University of Warwick (2012).

8. V. Knap, D.-I. Stroe, M. Swierczynski, R. Teodorescu, and E. Schaltz, J. Electrochem. Soc., 163, A911-A916 (2016).

9. Z. Deng, Z. Zhang, Y. Lai, J. Liu, J. Li, and Y. Liu, J. Electrochem. Soc., 160, A553-A558 (2013). 
2016-12-07

\section{Significance of the capacity recovery effect in pouch lithium-sulfur battery cells}

Knap, Vaclav

The Electrochemical Society

V. Knap, T. Zhang, D-I. Stroe et al., Significance of the capacity recovery effect in pouch lithium-sulfur battery cells. ECS Transactions, 2016, Volume 74, Issue 1, pages 95-100 http://dx.doi.org/10.1149/07401.0095ecst

Downloaded from Cranfield Library Services E-Repository 